\title{
A methodological protocol for selecting and quantifying low-value prescribing practices in routinely collected data: an Australian case study
}

\author{
Jonathan Brett ${ }^{1}$, Adam G. Elshaug ${ }^{2}$, R. Sacha Bhatia ${ }^{3,4}$, Kelsey Chalmers², Tim Badgery-Parker ${ }^{2}$
} and Sallie-Anne Pearson ${ }^{1,2^{*}}$

\begin{abstract}
Background: Growing imperatives for safety, quality and responsible resource allocation have prompted renewed efforts to identify and quantify harmful or wasteful (low-value) medical practices such as test ordering, procedures and prescribing. Quantifying these practices at a population level using routinely collected health data allows us to understand the scale of low-value medical practices, measure practice change following specific interventions and prioritise policy decisions. To date, almost all research examining health care through the low-value lens has focused on medical services (tests and procedures) rather than on prescribing. The protocol described herein outlines a program of research funded by Australia's National Health and Medical Research Council to select and quantify low-value prescribing practices within Australian routinely collected health data.

Methods: We start by describing our process for identifying and cataloguing international low-value prescribing practices. We then outline our approach to translate these prescribing practices into indicators that can be applied to Australian routinely collected health data. Next, we detail methods of using Australian health data to quantify these prescribing practices (e.g. prevalence of low-value prescribing and related costs) and their downstream health consequences. We have approval from the necessary Australian state and commonwealth human research ethics and data access committees to undertake this work.

Discussion: The lack of systematic and transparent approaches to quantification of low-value practices in routinely collected data has been noted in recent reviews. Here, we present a methodology applied in the Australian context with the aim of demonstrating principles that can be applied across jurisdictions in order to harmonise international efforts to measure low-value prescribing. The outcomes of this research will be submitted to international peer-reviewed journals. Results will also be presented at national and international pharmacoepidemiology and health policy forums such that other jurisdictions have guidance to adapt this methodology.
\end{abstract}

Keywords: Routinely collected data, Prescribing, Medicines, Low-value care, Protocol

\footnotetext{
* Correspondence: sallie.pearson@unsw.edu.au

${ }^{1}$ Medicines Policy Research Unit, Centre for Big Data Research in Health, University of New South Wales, Level 1, AGSM Building (G27), Sydney, NSW 2052, Australia

${ }^{2}$ Menzies Centre for Health Policy, School of Public Health, University of Sydney, Level 6 The Hub, Charles Perkins Centre D17, Sydney, NSW 2006, Australia

Full list of author information is available at the end of the article
} International License (http://creativecommons.org/licenses/by/4.0/), which permits unrestricted use, distribution, and reproduction in any medium, provided you give appropriate credit to the original author(s) and the source, provide a link to the Creative Commons license, and indicate if changes were made. The Creative Commons Public Domain Dedication waiver (http://creativecommons.org/publicdomain/zero/1.0/) applies to the data made available in this article, unless otherwise stated. 


\section{Background}

There is a growing recognition in health care that less may actually be more and that safety and effectiveness may be compromised in the face of 'too much medicine' [1]. A recent US study examining regional variation in test ordering, procedures and prescribing found that up to $30 \%$ of practices are potentially harmful or wasteful [2]. Moreover, health care costs are spiralling due to the increasing volume of services and interventions for ageing populations and the introduction of new and emerging high-cost services and treatments. Low-value practices, including tests, procedures and prescribing that provide little or no benefit, may result in patient harm or wasted resources [3]. The process of identifying harmful or wasteful practices is not new, but the field has gathered renewed global momentum and publicity because of growing imperatives to maximise safety, effectiveness and benefits from health care investments [4]. There is a need to quantify nominated low-value practices at the population level in order to benchmark current activities, prioritise policy initiatives and measure changes resulting from policy interventions [5].

\section{Low-value prescribing practice nomination}

There have been numerous attempts to systematically nominate low-value or inappropriate prescribing practices. However, in many circumstances, a lack of provider and public consultation has led to feelings of top-down stewardship and rationing [6-8]. Physician-led, consensus-based guidelines such as the Beers and STOPP criteria $[9,10]$ have also been developed to identify potentially inappropriate prescribing practices, but these tend to focus on specific populations (such as the elderly). Recent campaigns conducted through the lens of 'low-value care' have coalesced into the creation of specialty-specific, national lists of lowvalue practices that are not necessarily restricted to specific sub-populations [3]. Since 2007, the UK's National Institute of Health and Care Excellence (NICE) has curated the 'do not do' guidance in association with the Cochrane Collaboration [6]. Similarly, the Choosing Wisely campaign is a grassroots physician-led program that was launched formally in 2012 in the USA and has since spread to Canada, Australia, Japan and Europe [11]. This program aspires to be a transparent and consistent process of speciality-wide consultation, in collaboration with patient representatives to nominate specialty-based 'top 5 lists' of low-value practices based on evidence [12] and shared values [7]. There are a number of other similar current initiatives detailing low-value practices within medicine such as the Royal Australasian College of Physicians' EVOLVE program, the British Medical Journal's Too Much Medicine series and the Journal of the American Medical Association's Less is More series [13-15].

\section{Low-value prescribing practice quantification}

The science of measuring low-value care is in its infancy. At the population level, quantification aims to determine the frequency of low-value practices as well as variation (geographical or by other factors), changes over time, associations with patient and provider characteristics and downstream health consequences and costs [5]. Recent advances in analytical methods and access to routinely collected health data have created possibilities for quantifying low-value care at the population level [16-18]. However, a major challenge is that many nominated practices and downstream consequences are finely nuanced and identify specific populations and clinical contexts that may not be recorded within routinely collected data [8].

There have been efforts to quantify harmful or wasteful prescribing using routinely collected data prior to the low-value movement [19], but attempts to systematically review the historical literature are challenging because of the lack of common key words and MeSH terms. Systematic reviews of pharmacoepidemiological studies undertaken using Australian and Nordic routine data collections found that studies quantifying harmful and/ or wasteful practices were in the minority $[20,21]$. Some examples include measuring the extent of potentially inappropriate medication use in the elderly [22, 23] and consensus-based lists of inappropriate psychotropic prescribing [24]. Following the evolution of low-value lists, there have been some efforts to benchmark low-value practice frequency and quantify the impact of the Choosing Wisely campaign using routine data collections. A recent systematic review of low-value measures identified a lack of transparency in translating practices into indicators and the need to validate any measures that are developed [25]. Studies to date have also tended to focus on low-value tests and procedures rather than on prescribing [26-28]. These studies use varying quantification approaches, thus limiting direct comparisons, and so, there is a need to standardise this methodology to enhance the global effort.

Therefore, the objectives of this research program are to detail methods for:

- Cataloguing nominated low-value prescribing practices from low-value lists published internationally. This process is ongoing and will be updated to incorporate additional practices appearing on international lists.

- Translating low-value prescribing practices into indicators that can be applied to Australian routine data collections.

- Quantifying the extent of low-value prescribing practices in Australia.

- Quantifying the downstream consequences of low-value prescribing practices in Australia. 
The principles outlined in this protocol paper are generalisable to research in other jurisdictions attempting to quantify low-value prescribing practices in their routine data collections.

\section{Methods}

\section{Cataloguing low-value prescribing practices}

We are creating a catalogue of low-value prescribing practices by extracting nominated practices from international Choosing Wisely lists. Table 1 summarises the number of prescribing practices within each list as of August 2016 (full list of all practices available on request from authors). Collectively, these Choosing Wisely lists contain 189 individual prescribing practices representing $22 \%$ of all medical practices within these lists. We will update our catalogue as these lists continue to grow and other countries become engaged with the Choosing Wisely campaign.

\section{Duplication of practices within and between lists}

After identifying specific practices from the lists, we will break them into component parts: medication(s), indication(s), population(s) of intended use and other specifications. This assists with subsequent translation to indicators as part of the measurement process (described below). After grouping practices according to these components, we will identify similarities both within (when the practice was nominated by two different specialty groups on the same jurisdictional list) and between jurisdictional lists. In some cases, the practices will be an exact duplication. For example, within the Choosing Wisely USA list, 'Don't use antipsychotics as first choice to treat behavioural and psychological symptoms of

Table 1 Number of prescribing practices by Choosing Wisely list as of August 2016

\begin{tabular}{lll}
\hline List & $\begin{array}{l}\text { Total number } \\
\text { practices within } \\
\text { list }^{\mathrm{a}}\end{array}$ & $\begin{array}{l}\text { Number (\%) prescribing } \\
\text { practices within list }\end{array}$ \\
\hline $\begin{array}{l}\text { Choosing Wisely } \\
\text { USA [64] }\end{array}$ & 421 & $86(20 \%)$ \\
$\begin{array}{l}\text { Choosing Wisely } \\
\text { Canada [65] } \\
\text { Choosing Wisely }\end{array}$ & 176 & $41(23 \%)$ \\
$\begin{array}{l}\text { Italy [66] } \\
\text { Choosing Wisely }\end{array}$ & 93 & $20(18 \%)$ \\
$\begin{array}{l}\text { Australia [67] } \\
\text { Choosing Wisely }\end{array}$ & 10 & $33(35 \%)$ \\
$\begin{array}{l}\text { International top } \\
10 \text { list [54] }\end{array}$ & $5(50 \%)$ \\
$\begin{array}{l}\text { Choosing Wisely } \\
\text { Netherlands [68] } \\
\text { Choosing Wisely } \\
\text { Switzerland [69] }\end{array}$ & 35 & $2(6 \%)$ \\
\hline
\end{tabular}

aprescribing practices may be represented more than once within and between lists dementia' is specified using identical wording within both the American Society for Geriatrics and the American Psychiatric Association top 5 lists. In other instances, lists will nominate the same practice but use different wording. We also anticipate that similar practices may only have some common components, often meaning that they are broader or narrower definitions of a low-value practice.

\section{Defining broader and narrower definitions of low-value practices}

Schwartz et al. [18] recognised that definitions of individual low-value practices vary within and across lists, meaning that broader and narrower definitions of similar practices may be measured to determine a range over which similar practices occur. The narrowest definition likely captures the most certain instances of low-value care but may exclude some cases classified as low value by other similar practices. In contrast, the broadest likely captures all instances but may also include some instances of care defined as appropriate by other similar practices. Where possible, we will identify broader and narrower definitions within lists of similar prescribing practices by identifying increasingly narrower definitions within the medicine component of the practice: medicine class, subclass or individual medicine. Within the indication component, we will specify organ-level disorders (broader) versus specific diagnoses (narrower). We will also identify prescribing practices with the same medicine and indication that specify subpopulations (e.g. by specifying an age range) and others that add caveats (within the 'other specifications' component) such as 'first-line treatment'. For groups of practices with shared components, we will order the components of each prescribing practice according to whether they are broader or narrower than the corresponding components of other practices within the group (illustrated in Fig. 1).

\section{Translating low-value prescribing practices into indicators applied to Australian routine data collections}

In this section, we first detail the study setting and the core characteristics of Australia's routinely collected data as they pertain to the development of prescribing indicators and quantification of low-value prescribing practices. Second, we will detail the methods we will use to translate low-value practices into indicators applied to these data collections.

\section{Australia's health care setting}

Australia has a publicly funded universal health care system entitling all Australian citizens and permanent residents to a range of subsidised health services. This includes free treatment in public hospitals (funded jointly by the commonwealth and state/territory governments) and subsidised treatment in private hospitals (funded jointly by the commonwealth and private health 


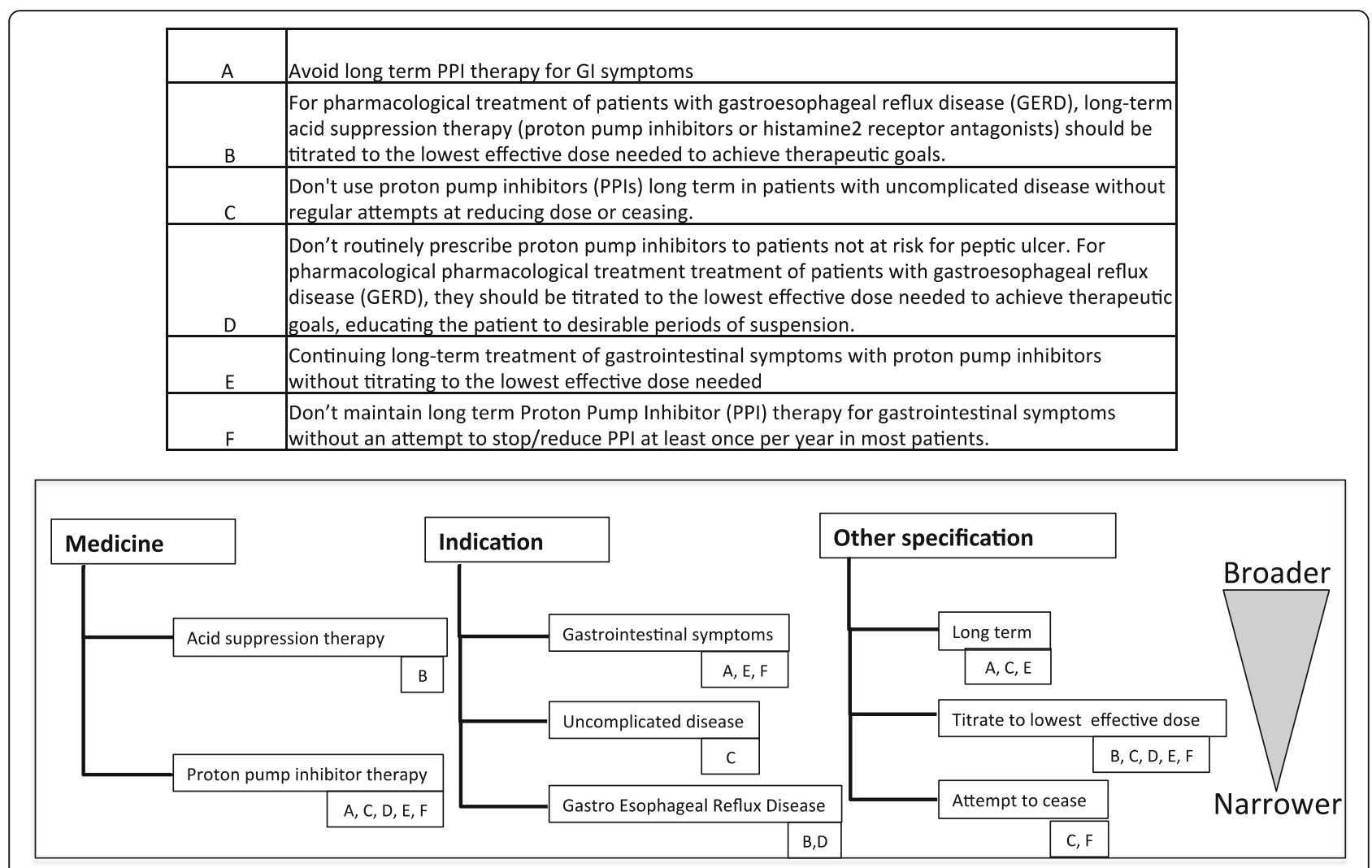

Fig. 1 Example of categorising prescribing practices into broader and narrower definitions

insurance). It also includes a range of subsidised outpatient services including consultations with medical and selected health care professionals (funded by the commonwealth's Medicare Benefits Scheme (MBS)) and medicines prescribed in the community and private hospitals and on discharge from some public hospitals (funded by the commonwealth's Pharmaceutical Benefits Scheme (PBS); medicines prescribed to public hospital inpatients are covered primarily by hospital budgets). In addition, the Australian Government Department of Veterans' Affairs (DVA) funds the health care of eligible veterans, war widows and widowers and their dependants. DVA clients are eligible to receive all health services and pharmaceuticals accessible to the general Australian population and, depending on their level of entitlement, additional DVA-approved services and pharmaceutical items. Historically, DVA data have been the primary data source for pharmacoepidemiological research in Australia as they are a sole payer and maintains custodianship over a wide variety of routine data collections including dispensing and medical service claims and hospitalisations.

\section{Australia's routine data collections}

We restrict our commentary in this section to data collections (either stand-alone or linked to each other) that have the capacity to track individual patients over time. These collections are summarised in Table 2.

There are two main sources of medicine data: (1) population-based pharmaceutical claims (payment) data captured after a prescribed medicine has been dispensed and subsidised under the PBS and (2) prescribing data captured at the point of care.

\section{Pharmaceutical Benefits Scheme data}

PBS claims will be the primary data source for the research program outlined in this protocol. Using dispensing claims data, we have the capacity to estimate temporal trends in dispensing as well as patterns of exposure (e.g. initiation, duration of use, dose and discontinuation) at the person level. PBS dispensing claims data also contain demographic details about the patient, such as age, gender and geographic location of residence, and also have the capacity to track prescriber behaviour through a unique prescriber identification number, although there is limited additional information about prescriber characteristics beyond their specialty of practice. PBS claims do not contain information such as the prescribed daily dose of a medicine or the indication for which a medication is prescribed. These features of PBS claims data are typical of dispensing claims data worldwide [29]. 
Table 2 Australian datasets (stand-alone or linked) available to quantify low-value prescribing practices

Dataset Variables of interest

Medicines prescribed in the community

Dispensing

Dataset: Pharmaceutical Benefits Scheme (PBS) dispensing

claims. Available 2005 to current

Custodian: Australian Government Department of Human Services

Prescribing

Dataset: Medicinelnsight. Available 2010 to current

Custodian: NPS MedicineWise

Datasets to which PBS dispensing data will be linked

Tests, procedures and outpatient consultations

Dataset: Medical Benefits Schedule (MBS) service claims.

Available 2004 to 2013

Custodian: Australian Government Department of Veterans Affairs

Hospitalisations

Dataset: NSW Admitted Patient Data Collection

Available July 2000 to current

Custodian: Director, Demand and Performance Evaluation Branch,

NSW Department of Health

Emergency department visits

Dataset: NSW Emergency Department Data Collection. Available

2005 to current

Custodian: Director, Demand and Performance Evaluation Branch,

NSW Department of Health

\section{Cancer notifications}

Dataset: NSW Central Cancer Registry. Available 1994 to current

Custodian: Central Cancer Registry NSW

Death data

Dataset: Registry of Births, Deaths and Marriages NSW. Available

1994 to current

Custodian: Registrar of Births, Deaths and Marriages NSW
Item number, date of prescribing and dispensing service, patient co-payment, cost to government, patient demographics (age, gender, location of residence mapped to Socioeconomic Index and Remoteness classifications), provider location (also mapped), fact of death

Prescribed medicines (date of prescribing; prescribed daily dose; indication for prescribing), test ordering (date of request, results), patient demographics (age, gender, location of residence mapped to Socioeconomic Index and Remoteness classifications), patient past medical and family history, referrals, management plans, immunisations, provider location (also mapped)

Item number, date of service, scheduled fee, provider charge, benefits paid, patient co-payment, patient demographics (age, gender, location of residence mapped to Socioeconomic Index and Remoteness classifications), provider location (also mapped)

Diagnostic and procedure codes, diagnosis-related groups (admission costs), hospital type, separation date and status, geographic location of patient residence and hospital; patient demographics (age, gender, place of residence)

Triage category, diagnostic codes; mode of arrival; hospital type, geographic location of patient residence and hospital, separation date and status, patient demographics (age, gender, place of residence)

Date of new cancer diagnosis, type of cancer, stage at diagnosis

Cause of death, place of death, date of death, decedent demographics (age, gender, place of usual residence)

\section{Prescribing data}

Currently, Australia does not have any population-based, routinely collected prescribing datasets. Rather, data can be obtained and complied from individual community medical practices or public hospitals. Most general practices in Australia use electronic prescribing platforms that have the capacity to capture prescribing of PBS-listed and other medicines, but this is not necessarily the case in specialty practice [30, 31]. Medicines prescribed to public hospital inpatients are funded primarily by hospital budgets at the state level, and electronic prescribing systems have become increasingly commonplace in Australian public hospitals. Significant challenges currently exist in Australia in terms of unifying prescribing data across more than one hospital network and multiple community practices, due in part to the high degree of variability in software used and local governance arrangements. In addition, the quality and comprehensiveness of prescribing data is highly variable and dependent on end-user inputs.

However, one of the most promising prescribing datasets that has the potential for use in our study is from the MedicineInsight initiative [32], an Australian Governmentfunded programme that aims to increase the understanding of prescribing behaviour in Australian general practice. Comprising approximately 500 practices throughout Australia, it is the first large-scale, longitudinal general practice data program in Australia that extracts deidentified patient health records from existing general practice software. Compared with PBS dispensing data, these data contain greater depth of clinical information about the practice, the prescriber and the patient, as well as the clinical indication(s) for which the medicine is prescribed, tests are ordered and referrals are made [33]. However, because this is a relatively new program and general practice recruitment is ongoing, it is unclear how nationallyrepresentative the data are currently.

\section{Prescribed medicine data linked to other routinely collected datasets}

We will utilise a range of population-based data comprising PBS dispensing claims linked to other routine collections held by the Commonwealth of Australia (Medicare service claims) and the individual states (hospitalisations, emergency department visits, cancer notification, fact and cause of death data) to implement our proposed research. For the purposes of the specific 
quantification, the example outlined below will use the DVA linked dataset, a data source that has been used extensively in Australian pharmacoepidemiological studies [20]. However, for future work quantifying other prescribing practices, we may use other linked datasets. Table 2 outlines the population-based collections that have been linked to dispensing claims data within the DVA dataset. The advantage of such linked collections is that they provide a more detailed picture of an individual's health that can be helpful when attempting to isolate low-value practices. Linked data also enhance the capacity to establish the downstream consequences of low-value care and to account for confounders when performing outcome studies. While it is technically feasible to link prescribed medicine data at the individual level to other routine data collections, currently it will only yield data for a (non-representative or biased) sample of hospital or community settings in Australia. Efforts to link pathology results data to pharmaceutical dispensing claims on a national level are limited in Australia due to the multitude of pathology services, and as with integrating hospital prescribing nationally, there is a high degree of variability in software used and local governance arrangements.

\section{Indicator development and application}

We will construct indicators by matching the component parts of prescribing practices to variables within routinely collected data. The collection of variables corresponding to a prescribing practice will represent the practice indicator. For the purposes of illustration, we have selected nine practices identified from the Choosing Wisely lists (Table 3 ). These practices represent the broadest definitions within their groups of similar practices. Practices that are similar but narrower are listed in Additional file 1: Table S1. In the simplest case, the components of the practice 'don't prescribe benzodiazepines to elderly people' are benzodiazepines (medicine) and elderly people (population). Medicine and age are both variables within pharmaceutical dispensing claims data, and so, the indicator would consist of exposure to benzodiazepines and a defined age group and this practice could be measured in stand-alone PBS data.

When we are unable to directly translate prescribing practices into indicators, as they contain components that do not have equivalent variables within existing datasets, we will create proxy measures from existing variables. For instance, the practice 'avoid antipsychotics in dementia' is comprised of antipsychotics (medicines) and dementia (indication). If attempting to quantify this within dispensing claims data alone, where indication for prescribing and information on comorbidities are generally not available, we will create proxy markers for disease states (such as the prior
Table 3 Low-value prescribing practice case examples and Choosing Wisely list origin

\begin{tabular}{|c|c|c|}
\hline $\begin{array}{l}\text { Prescribing practice } \\
\text { example number }\end{array}$ & Practice & $\begin{array}{l}\text { Choosing } \\
\text { Wisely list }\end{array}$ \\
\hline 1 & $\begin{array}{l}\text { Avoid prescribing antibiotics } \\
\text { for upper respiratory infections }\end{array}$ & USA \\
\hline 2 & $\begin{array}{l}\text { Don't use benzodiazepines in } \\
\text { the elderly }\end{array}$ & International \\
\hline 3 & $\begin{array}{l}\text { Avoid long-term PPI therapy } \\
\text { for Gl symptoms }\end{array}$ & International \\
\hline 4 & Avoid antipsychotics for dementia & International \\
\hline 5 & $\begin{array}{l}\text { Do not use antibiotics in } \\
\text { asymptomatic bacteriuria }\end{array}$ & Australia \\
\hline 6 & $\begin{array}{l}\text { Don't recommend the regular } \\
\text { use of oral non-steroidal anti- } \\
\text { inflammatory medicines (NSAIDs) } \\
\text { in older people }\end{array}$ & Australia \\
\hline 7 & $\begin{array}{l}\text { Don't prescribe testosterone } \\
\text { therapy unless there is evidence } \\
\text { of proven testosterone deficiency }\end{array}$ & Australia \\
\hline 8 & $\begin{array}{l}\text { Don't initiate and continue } \\
\text { medicines for primary prevention } \\
\text { in individuals who have a limited } \\
\text { life expectancy }\end{array}$ & Australia \\
\hline 9 & $\begin{array}{l}\text { Don't routinely prescribe two or } \\
\text { more antipsychotic medications } \\
\text { concurrently }\end{array}$ & USA \\
\hline
\end{tabular}

dispensing of an anti-dementia medication). Similarly, for the practice 'Don't routinely prescribe two or more antipsychotic medications concurrently', we will use a proxy for medication concomitance within dispensing claims data as the intended duration of exposure following a medication dispensing is not known. The uncertainty introduced by these proxies can be somewhat mitigated if dispensing claims are linked to other data sources such as hospitalisation data, which contain hospitalisation-related diagnostic codes. While the degree of uncertainty introduced by these proxy measures is difficult to quantify, we will perform sensitivity analyses, such as varying the boundaries of decision rules within these proxy variables to observe the magnitude of the effect on practice quantification. Prescribing data can also be used if an indication is required and cannot be inferred from dispensing claims or hospitalisations. However, as previously discussed, the quality of diagnostic information is end-user dependent and we aim to focus on dispensing claims for this Australian analysis as the generalisability of findings from existing Australian prescribing data are unclear. Furthermore, we do not currently have access to dispensing claims linked to pathology data. However, in the interests of this protocol being adaptable to an international audience, we have included prescribing and pathology data at the stage of indicator translation. Table 4 illustrates 


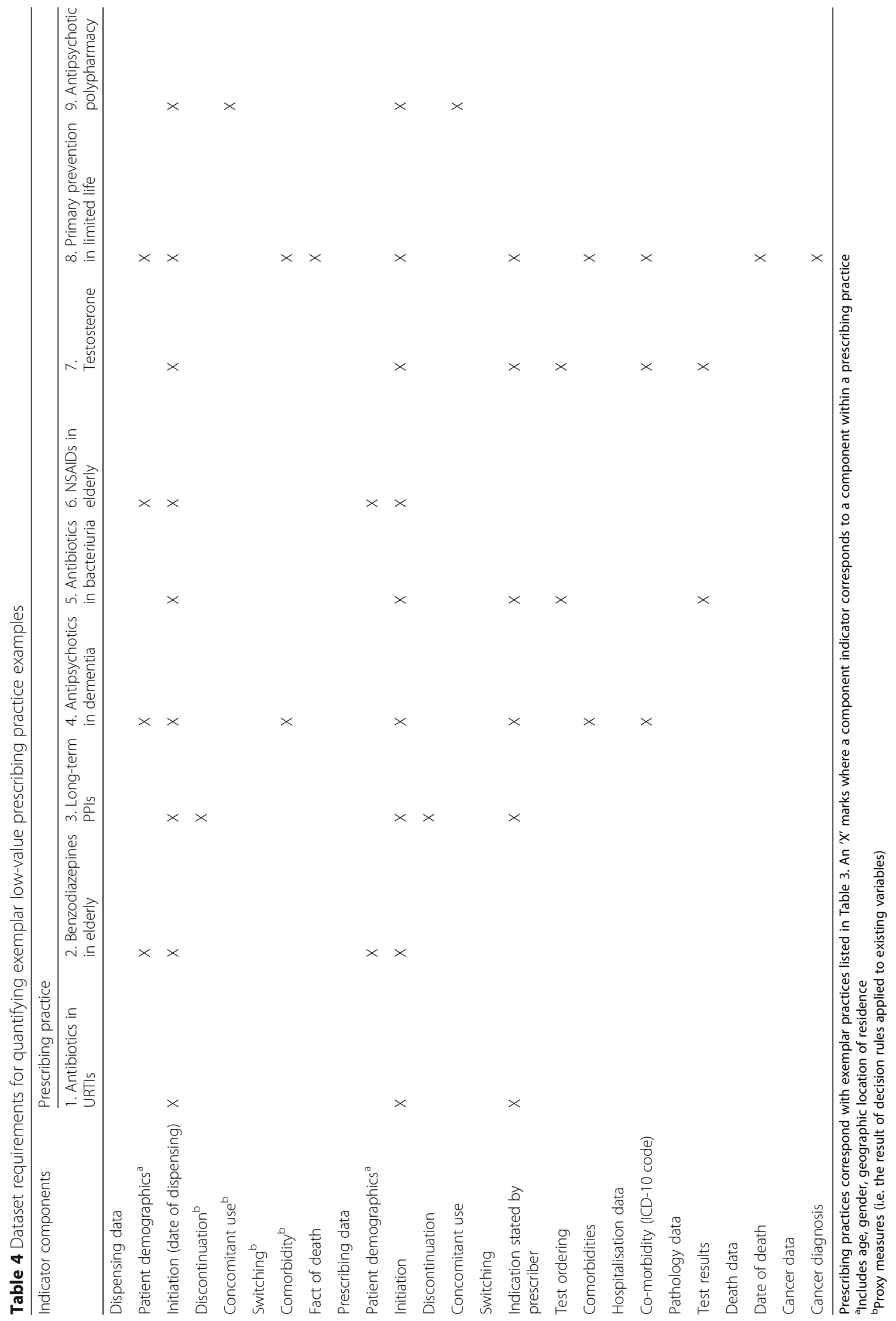


how the nine exemplar prescribing practices (Table 3) can be translated into their corresponding indicators in Australian data.

There may often be several approaches to quantifying the same practice that vary in accuracy and precision by using different indicators comprised of a range of different variables, potentially from different data sources. When adapting these methods to other jurisdictions, the most appropriate approach depends on local data access, coverage and analytical expertise.

\section{Quantifying the extent of a low-value prescribing practice in Australia}

Our research program will undertake a series of observational cohort studies to quantify the extent of low-value prescribing in Australia. In this section, we describe specific quantification methodology for one low-value prescribing practice, 'Don't routinely prescribe two or more antipsychotic medications concurrently', specified within the USA Choosing Wisely list and hereafter referred to as antipsychotic polypharmacy. The general approach outlined here will apply to all of the low-value prescribing practices detailed in this protocol when we utilise PBS claims linked with other routine collections.

\section{Cohort definition}

We will use stand-alone Pharmaceutical Benefits Scheme (PBS) dispensing claims from 1 March 2005 to the most contemporary available (currently January 2017) to perform our analysis [34] (Table 2). We will identify people dispensed antipsychotics (WHO Anatomical Therapeutic Classification codes NO5A) and define antipsychotic concomitance as overlapping courses of two or more non-like antipsychotics for 60 days or more. However, we will also perform sensitivity analyses using 14- and 90-day cutoffs. PBS dispensing claims contain only the date medicines are dispensed, and so, to identify courses of antipsychotics, we will first estimate the duration of exposure (in days) following a single dispensing of each antipsychotic medicine, hereafter referred to as the estimated period of exposure (EPE). For each antipsychotic, we will measure the intervals between dispensings of the same medicine in individuals who have more than one dispensing of that medicine over the study period. The EPE for a medicine will then be calculated as the number of days in which $75 \%$ of people have received a subsequent dispensing of the same medicine. A course of a given antipsychotic medicine will be defined as the period in which there are serial dispensings of the same medicine and the EPEs of these dispensings overlap. This method has been applied previously to psychotropics and accounts for variability in dispensing due to adherence, dose changes and seasonality $[35,36]$.

\section{Practice quantification}

We will quantify the extent of and variability in antipsychotic polypharmacy. Specifically, we will determine:

(a) The number of patients experiencing antipsychotic polypharmacy expressed as a proportion of all people treated with antipsychotics. We will calculate the number of episodes of antipsychotic polypharmacy as well as the number (mean, median, interquartile range (IQR) and maximum and minimum) of episodes per patient. We will describe this cohort according to age, gender, location of residence, rurality and socioeconomic stratum.

(b) The number of prescribers delivering low-value prescribing expressed as a proportion of the total number of prescribers in the study cohort. We will also calculate the number (means, medians, interquartile ranges and maxima and minima) of episodes of antipsychotic polypharmacy per prescriber and whether antipsychotic polypharmacy was initiated or continued by that prescriber. We will then describe these prescribers according to available prescriber variables. Within the PBS dispensing claims, this is limited to speciality, but in other datasets, in particular prescribing data, additional variables include number of years in practice and rurality and socioeconomic characteristics of practice location.

(c) Variation in low-value care. We will examine the variation in antipsychotic polypharmacy by stratifying analyses according to prescriber location (rurality and socioeconomic stratum of practice location) and patient characteristics (age, gender, location of residence, rurality and socioeconomic stratum) using established methodology [37, 38]. We will also use Poisson, negative binomial and/or multilevel regression models to determine the influence of these patient and provider characteristics on antipsychotic polypharmacy counts [39].

(d) Changes over time and following policy intervention. Analyses a. to c. will be annualised to demonstrate changes over time. We will calculate the prevalence and incidence of antipsychotic polypharmacy where prevalent use is defined as persons with at least one episode of antipsychotic polypharmacy within a given calendar year and incident (new) use is defined as persons with an episode of antipsychotic polypharmacy within a given calendar year and no episode of antipsychotic polypharmacy in the previous 12 months. Additionally, we will use interrupted time series analyses as previously applied to PBS dispensing claims $[40,41]$ to examine changes in antipsychotic 
polypharmacy before and after interventions that may impact on this practice. An example of such an intervention in Australia that will be investigated is the change in the prescribing restrictions for antipsychotics that had required telephone authority but moved to streamlined authority on 1 July 2007 [42]. With fewer barriers to prescribing, this may have increased antipsychotic polypharmacy. Given concerns about antipsychotic polypharmacy, this may prompt a review of prescribing authority policy. We will use an autoregressive integrated moving average (ARIMA) approach using the Box-Jenkins method which will be used to model subsequent time series while accounting for seasonal variability, as well as long-term trends and autocorrelation [43].

\section{Quantification of downstream consequences}

Here, we start with a discussion regarding the general concepts involved in quantifying downstream consequences of low-value prescribing practices within routinely collected data and then return to detail how these are applied to quantify the downstream consequences of antipsychotic polypharmacy. Low-value prescribing practices may expose individuals to additional, unnecessary risks, and investigating the measurable harms of these practices is an important research endevour. Adverse drug events (ADEs) known to be associated with each practice will be identified from the literature, and valid indicators corresponding to these ADEs subsequent to an index dispensing will be identified within routinely collected health datasets. These may be medicine-based ADE indicators such as the dispensing of a medicine known to be associated with an ADE or diagnostic indicators, such as ICD-10 codes in hospitalisation data or the International Classification of Primary Care (second edition) classification (ICPC) system used in general practice. ADE [44] and disease state [45] indicators have been validated in Australian veterans' affairs data. While disease state indicators were validated to measure comorbidity, they also have clinical face validity as ADE markers. Generally, these ADE indicators have been shown to have good specificity but lower sensitivity when compared to the gold standards of record review and self-reported health surveys [44-46]. The other limitation is that they vary in how accurately they reflect the actual timing of onset of the ADE. Additional file 2: Table S2 identifies the potential ADEs associated with each prescribing practice example in Table 3, and Additional file 3: Table S3 details the potential medicine and diagnostic-based indicators of these adverse drug events.

Antipsychotic polypharmacy has been associated with increased risk of parkinsonism, dyslipidaemia, cardio and cerebrovascular disease, diabetes, cognitive impairment and sudden cardiac death (Additional file 2: Table S2)
[47].Using the same study cohort and study period defined above, we will use prescription sequence analysis [44] to determine the association of antipsychotic polypharmacy with an incident dispensing (i.e. new dispensing with no dispensings in the 12 months prior) of these ADE medicine-based indicators. The crude sequence ratio will be calculated by dividing the number of people with an incident dispensing of an ADE indicator medicine in a 12month post-exposure period (after the start of antipsychotic polypharmacy) with the number of people with incident dispensing of an ADE indicator medicine in a 12-month pre-exposure period (before the start of antipsychotic polypharmacy). We will perform sensitivity analyses, varying the length of pre- and post-exposure periods (3, 6 and 18 months). We will estimate the probability that the ADE medicine is dispensed first, in the absence of any causal relationship, adjusting for the null-effect sequence ratio which accounts for underlying changes in the incidence of dispensing of the ADE medicine (see [48] for details and formula). We will then calculate the adjusted sequence ratio (ASR) by dividing the crude by the null-effect ratio, and 95\% confidence intervals will be calculated for this value. A signal is considered to be present when the lower limit of the $95 \% \mathrm{CI}$ is one or more.

When dispensing claims data are linked to other health databases, a greater range of outcome variables are available. In these cases, cohort and case-control study designs with appropriate regression analyses [49] can be used to quantify the association between an index prescribing event and an ADE using an appropriate ADE indicator while adjusting for patient and prescriber factors. Choice of study design will depend on the known natural history of the $\mathrm{ADE}$, its temporal relationship to the low-value practice exposure and the availability of a control group.

In this specific example, we will apply a self-controlled case series analysis to determine whether antipsychotic polypharmacy leads to increased risk of acute vascular disease. This will require linkage of PBS dispensing claims and hospitalisation data, and for this purpose, we have access to Department of Veterans Affairs (DVA) linked dataset (Table 2). Self-controlled case series analysis effectively measures incidence rate ratios of relatively acute ADEs while controlling for measured and unmeasured fixed patient confounders [50,51]. We will use PBS dispensing claims linked to hospitalisation data for DVA beneficiaries from 1 January 2006 and 31 December 2015 but results will be reported from 1 January 2007 to allow for a 1-year look-back when identifying incident antipsychotic polypharmacy. Patients will be eligible for inclusion if they have hospital diagnostic codes related to acute ischemic heart disease (ICD-10: I20-24) and stroke (ICD-10: I60-I64) during the study period and if they had been full entitlement holders (eligible for 
all health services) for at least 12 months at the time of diagnosis. Incident antipsychotic polypharmacy (i.e. new episodes with no episodes in the 12 months prior) will be identified for these individuals during this period. The end of antipsychotic polypharmacy will be defined as one EPE after the last concomitant antipsychotic dispensing. As previously described [52] exposed persontime will be divided into 1,2 to 4 and 5 to 12 weeks after the start of antipsychotic polypharmacy. A pre-exposure risk period will also be divided into 1,2 to 4 and 5 to 12 weeks prior to the start of antipsychotic polypharmacy, and this will be considered separately from non-exposed time to account for confounding by indication (i.e. in the case that polypharmacy was initiated as a consequence of hospitalisation). Some individuals will have no exposure to antipsychotic polypharmacy for the entire study period but will be included in the analysis to contribute information about possible confounders. The count of outcomes in each of these exposure risk periods will be compared with the count of outcomes in non-exposed periods. Conditional Poisson regression will be used to calculate incidence rate ratios for the two primary outcomes (hospitalisation with ischemic heart disease or stroke) adjusting for age, gender and calendar year.

\section{Incremental costs}

We will use dispensing claims data to calculate the medication costs to the payer (government or private health care fund) and out-of-pocket costs to the patient for psychotropic polypharmacy and compare these costs to antipsychotic monotherapy. Excess hospitalisations with cardiovascular diseases and stroke will be extrapolated from incidence rate ratios, and excess costs will be calculated using length of stay and hospital diagnosisrelated groups (DRGs) [53].

\section{Ethics}

The Population Health Sciences Research Ethics Committee has granted approval for our overall program of research (Approval Numbers 2013/11/494 and 2014/06/539).

\section{Consent and privacy considerations}

A combination of commonwealth, state, hospital and privately held data will be used as part of this program of work.

The use and disclosure of commonwealth and state data are governed under the Privacy Act 1988 and Health Records and Information Privacy (HRIP) Act 2003, respectively. We were able to access this data with a waiver of consent as under this legislation, it is not required if (1) it is impracticable to gain consent and (2) the use is in accordance with the section 95A guidelines (which provides a process to resolve the conflict that may arise between the public interest in privacy and the public interest in medical research).

We will minimise the risk to personal privacy by ensuring data are stored securely within the Centre for Big Data Research located at the University of New South Wales; only researchers involved in data analysis will have access to the data and that researchers do not have access to identified data or any means of reidentifying individuals within the data. Finally, all data will be presented in aggregated form only, and potentially identifiable information will not be published. We will suppress data with small cell sizes.

\section{Dissemination}

The outcomes of this research will be submitted to international peer-reviewed journals; in particular general medical, health policy and pharmacoepidemiology journals. Furthermore, results will be presented at national and international pharmacoepidemiology, health policy and Choosing Wisely forums. Direct access to the data and analytical files to other individuals or authorities is not permitted without the express permission of the approving human research ethics committees and data custodians.

\section{Limitations}

The potential limitations of this programme of research relate to low-value lists themselves and the application of these practices for quantification in routine data collections. The Choosing Wisely campaign has been criticised for the inclusion of low-impact, low-value practices, putatively because of conflict of interest with revenue streams for specific specialist groups [8]. However, this appears to be the exception rather than the rule [8], and one may argue that this is not likely to be the case for prescribing that typically does not generate income streams for physicians. The process of practice translation into indicators highlights limitations of using routine data collections to quantify prescribing practices such as inferences and things that cannot be easily measured. This is one consequence of lists that have been generated without measurement necessarily in mind and often results from lack of clinical information within routine data collections. This can also lead to substantial unmeasured confounding when attempting to identify associations between low-value prescribing practices and downstream ADEs. Using self-controlled study designs can help mitigate this unmeasured confounding. There is a need to validate more ADE indicators within Australian datasets using either medical record review or selfreported health surveys as gold standards. Issues such as data coverage and data access also limit generalisability of results. 


\section{Discussion}

The presence of prescribing practices in international Choosing Wisely initiatives clearly indicates there is cause for concern regarding low-value prescribing. However, to date there have been no systematic efforts to quantify these practices at a jurisdictional level. Furthermore, there have been no efforts to systematically determine the downstream consequences and associated costs of these low-value prescribing practices.

We noted similarities in prescribing practices within and between jurisdictional lists and that similar practices could be grouped (mostly according to medication and indication) during our first cataloguing process. Identifying groups of similar practices is one approach to identify priority areas for practice measurement: large groups of similar practices may represent shared areas of concern. They also represent opportunities to harmonise low-value practice definitions at jurisdictional and international levels by agreeing on a common list of practices to endorse. This would greatly assist quantification as within and between jurisdictional variability in low-value practice frequency could then be more easily measured and Choosing Wisely International has already started this process [54]. Until there is a common international list, identifying and quantifying broader and narrower definitions of similar practices allows a range over which similar practices occur to be identified. This method has been applied to quantifying tests and procedures [18]. However, compared to low-value tests and procedures, identifying the narrowest definitions of prescribing practices can be more challenging due to the greater and more varied number of components that prescribing practices are composed of.

As noted previously in our introduction, there are a number of other low-value lists such as the National Institute for Health and Care Excellence (NICE) 'do not do' list, the Royal Australasian College of Physicians EVOLVE project [11] and practices highlighted within JAMA Internal Medicine's and BMJ's 'less is more' and 'too much medicine' series, respectively. For the purposes of this protocol, we have focused exclusively on Choosing Wisely, but we intend on cataloguing all practices with other lists as they become available. It is important to note that many of the items identified within the Choosing Wisely lists are also present in these other lists.

Quantification efforts using routinely collected health data specifically assist in benchmarking population levels and costs of low-value practices as well as evaluating the impact of ensuing policy and practice decisions [5]. These quantification efforts may also directly facilitate behaviour change by providing methods of non-punitive audit and feedback to prescribers and patients [22].

Although Australian data has been used as an exemplar, it is our intent that these methods can be used by other jurisdictions as a standard framework to continue to prioritise and quantify prescribing practices at a jurisdictional level.

\section{Additional files}

Additional file 1: Table S1. Low-value prescribing practice case examples with broadest (highlighted) and narrower definitions along with Choosing Wisely list origin. (DOCX 105 kb)

Additional file 2: Table S2. Adverse drug events related to prescribing practice case examples as identified from the literature [47, 50, 55-63]. (DOCX 76 kb)

Additional file 3: Table S3. Pharmacy- and hospitalisation-based indicators of adverse drug events/diseases states identified in Additional file 2: Table S2 [44, 45]. (DOCX 96 kb)

\section{Acknowledgements}

This research was supported by the National Health and Medical Research Council (NHMRC) Centre for Research Excellence in Medicines and Ageing and program grant. Jonathan Brett is also funded by an NHMRC

postgraduate award.

\section{Funding}

This work is supported by an NHMRC Project Grant (APPID:1 109626), the NHMRC Centre for Research Excellence in Medicines and Ageing (APPID:1060407), an NHMRC Postgraduate Scholarship (APPID: 1094304). AGE receives salary support as the HCF Research Foundation principal research fellow and holds a grant from the Commonwealth Fund. KC and TBP receive salary support via a doctoral scholarship from the Capital Markets

Cooperative Research Centre-Health Market Quality Program.

\section{Availability of data and materials}

Please contact author for data requests.

\section{Authors' contributions}

$J B, R S B, K C, T B P, A G E$ and SP drafted and approved the final manuscript. JB catalogued the low-value prescribing practices.

\section{Competing interests}

AGE is a ministerial appointee to the (Australian) Medicare Benefits Schedule (MBS) Review Taskforce. He receives consulting/sitting fees from Cancer Australia, the Capital Markets Cooperative Research Centre-Health Market Quality Program, NPS MedicineWise (facilitator of Choosing Wisely Australia), the Royal Australasian College of Physicians (facilitator of the EVOLVE program) and the Australian Commission on Safety and Quality in Health Care. SP is a member of the Drug Utilization Sub-Committee of the Pharmaceutical Benefits Advisory Committee (PBAC). The views presented in this paper are those of the authors and do not reflect those of any of the entities with which they are affiliated.

\section{Consent for publication}

Not applicable.

Ethics approval and consent to participate

The Population Health Sciences Research Ethics Committee has granted approval for our overall program of research (Approval Numbers 2013/11/494 and 2014/06/539). A waiver of consent was granted as part of this research.

\section{Publisher's Note}

Springer Nature remains neutral with regard to jurisdictional claims in published maps and institutional affiliations.

\section{Author details}

${ }^{1}$ Medicines Policy Research Unit, Centre for Big Data Research in Health, University of New South Wales, Level 1, AGSM Building (G27), Sydney, NSW 2052, Australia. ${ }^{2}$ Menzies Centre for Health Policy, School of Public Health, University of Sydney, Level 6 The Hub, Charles Perkins Centre D17, Sydney, 
NSW 2006, Australia. ${ }^{3}$ Department of Medicine, University of Toronto, Toronto, Canada. Institute for Health System Solutions and Virtual Care, Women's College Hospital, Toronto, Canada.

\section{Received: 18 January 2017 Accepted: 18 April 2017}

Published online: 03 May 2017

\section{References}

1. Glasziou P, Moynihan R, Richards T, Godlee F. Too much medicine; too little care. Br Med J. 2013;347.

2. Fisher ES, Bynum JP, Skinner JS. Slowing the growth of health care costs—lessons from regional variation. N Engl J Med. 2009;360(9):849-52.

3. Elshaug AG, McWilliams JM, Landon BE. The value of low-value lists. JAMA. 2013;309(8):775-6.

4. Malhotra A, Maughan D, Ansell J, Lehman R, Henderson A, Gray M, et al. Choosing Wisely in the UK: the Academy of Medical Royal Colleges' initiative to reduce the harms of too much medicine. Br Med J. 2015:350:h2308.

5. Bhatia RS, Levinson W, Shortt S, Pendrith C, Fric-Shamji E, Kallewaard M, et al. Measuring the effect of Choosing Wisely: an integrated framework to assess campaign impact on low-value care. British Med J Qual Saf. 2015; 24(8):523-31.

6. Garner S, Littlejohns P. Disinvestment from low value clinical interventions: NICEly done? Br Med J. 2011;343:d4519.

7. Brody H. Medicine's ethical responsibility for health care reform - the top five list. N Engl J Med. 2010;362(4):283-5.

8. Morden NE, Colla CH, Sequist TD, Rosenthal MB. Choosing wisely-the politics and economics of labeling low-value services. N Engl J Med. 2014; 370(7):589-92.

9. Campanelli CM. American Geriatrics Society updated Beers Criteria for potentially inappropriate medication use in older adults: the American Geriatrics Society 2012 Beers Criteria Update Expert Panel. J Am Geriatr Soc. 2012;60(4):616

10. Gallagher P, Ryan C, Byrne S, Kennedy J, O'Mahony D. STOPP (Screening Tool of Older Person's Prescriptions) and START (Screening Tool to Alert doctors to Right Treatment). Consensus validation. Int J Clin Pharmacol Ther. 2008;46(2):72-83

11. Levinson W, Kallewaard M, Bhatia RS, Wolfson D, Shortt S, Kerr EA, et al. 'Choosing Wisely': a growing international campaign. BMJ Quality \& Safety. 2014:bmjas-2014-003821.

12. Gliwa C, Pearson SD. Evidentiary rationales for the Choosing Wisely top 5 lists. JAMA. 2014;311(14):1443-4.

13. Soon J, Buchbinder R, Close J, Hill C, Allan S, Turnour C. Identifying lowvalue care: the Royal Australasian College of Physicians' EVOLVE initiative. Med J Aust. 2016:204(5):180.

14. JAMA Internal Medicine. Less Is More Collection. Available from: http:// jamanetwork.com/collections/6017/less-is-more. Accessed Jan 2017.

15. Journal BM. Too Much Medicine. Available from: http://www.bmj.com/toomuch-medicine. Accessed Jan 2017

16. Kelman CW, Pearson S, Day RO, Holman CDJ, Kliewer EV, Henry DA. Evaluating medicines: let's use all the evidence. Med J Aust. 2007:186(5):249.

17. Collins B. Big Data and health economics: strengths, weaknesses, opportunities and threats. PharmacoEconomics. 2015:1-6

18. Schwartz AL, Landon BE, Elshaug AG, Chernew ME, McWilliams JM. Measuring low-value care in Medicare. JAMA Intern Med. 2014;174(7):1067-76.

19. Chan KS, Chang E, Nassery N, Chang H-Y, Segal JB. The state of overuse measurement: a critical review. Med Care Res Rev. 2013;70(5):473-96.

20. Pearson SA, Pesa N, Langton JM, Drew A, Faedo M, Robertson J. Studies using Australia's Pharmaceutical Benefits Scheme data for pharmacoepidemiological research: a systematic review of the published literature (1987-2013). Pharmacoepidemiol Drug Saf. 2015;24(5):447-55.

21. Wettermark B, Zoega H, Furu K, Korhonen M, Hallas J, Nørgaard M, et al. The Nordic prescription databases as a resource for pharmacoepidemiological research —a literature review. Pharmacoepidemiol Drug Saf. 2013;22(7):691-9.

22. Roughead E, Anderson B, Gilbert AL. Potentially inappropriate prescribing among Australian veterans and war widows/widowers. Intern Med J. 2007;37(6):402-5.

23. Cahir C, Fahey T, Teeling M, Teljeur C, Feely J, Bennett K. Potentially inappropriate prescribing and cost outcomes for older people: a national population study. Br J Clin Pharmacol. 2010;69(5):543-52.

24. Essock SM, Covell NH, Leckman-Westin E, Lieberman JA, Sederer LI, Kealey E, et al. Identifying clinically questionable psychotropic prescribing practices for Medicaid recipients in New York state. Psychiatr Serv. 2009;60(12):1595-602.
25. de Vries EF, Struijs JN, Heijink R, Hendrikx RJ, Baan CA. Are low-value care measures up to the task? A systematic review of the literature. BMC Health Serv Res. 2016;16(1):1.

26. Rosenberg A, Agiro A, Gottlieb M, Barron J, Brady P, Liu Y, et al. Early trends among seven recommendations from the Choosing Wisely campaign. JAMA Intern Med. 2015;175(12):1913-20.

27. Fleming-Dutra KE, Hersh AL, Shapiro DJ, Bartoces M, Enns EA, File TM, et al. Prevalence of inappropriate antibiotic prescriptions among US ambulatory care visits, 2010-2011. JAMA. 2016;315(17):1864-73.

28. Schwartz AL, Zaslavsky AM, Landon BE, Chernew ME, McWilliams JM. Low-value service use in provider organizations. Health Serv Res. 2016.

29. Strom BL. Pharmacoepidemiology: John Wiley \& Sons; 2006

30. Reeve J, Sweidan M. Setting a standard for electronic prescribing systems. Aust Prescr. 2011;34(1):2.

31. Australian Government Department of Health and Aging. The eHealth readiness of Australia's medical specialists. 2011.

32. National Prescribing Service. What is Medicine Insight? 2015. Available from: http://www.nps.org.au/health-professionals/medicineinsight. Accessed Jan 2017

33. DeVoe JE, Gold R, McIntire P, Puro J, Chauvie S, Gallia CA. Electronic health records vs Medicaid claims: completeness of diabetes preventive care data in community health centers. Ann Fam Med. 2011;9(4):351-8.

34. Mellish L, Karanges EA, Litchfield MJ, Schaffer AL, Blanch B, Daniels BJ, et al. The Australian Pharmaceutical Benefits Scheme data collection: a practical guide for researchers. BMC Res Notes. 2015;8(1):634.

35. Leach MJ, Pratt NL, Roughead EE. Psychoactive medicine use and the risk of hip fracture in older people: a case-crossover study. Pharmacoepidemiol Drug Saf. 2015.

36. Pratt NL, Ramsay EN, Ellett LMK, Nguyen TA, Barratt JD, Roughead EE. Association between use of multiple psychoactive medicines and hospitalization for falls: retrospective analysis of a large healthcare claim database. Drug Saf. 2014;37(7):529-35.

37. Leape LL, Park RE, Solomon DH, Chassin MR, Kosecoff J, Brook RH. Does inappropriate use explain small-area variations in the use of health care services? JAMA. 1990;263(5):669-72.

38. Care. ACoSaQiH. Australian Atlas of Healthcare Variation 2016. Available from: http://www.safetyandquality.gov.au/atlas/. Accessed Jan 2017.

39. Merlo J, Chaix B, Ohlsson H, Beckman A, Johnell K, Hjerpe P, et al. A brief conceptual tutorial of multilevel analysis in social epidemiology: using measures of clustering in multilevel logistic regression to investigate contextual phenomena. J Epidemiol Community Health. 2006:60(4):290-7.

40. Lagarde M. How to do (or not to do)... Assessing the impact of a policy change with routine longitudinal data. Health Policy Plan. 2012;27(1):76-83.

41. Schaffer AL, Buckley NA, Dobbins TA, Banks E, Pearson S-A. The crux of the matter: did the $A B C^{\prime}$ 's catalyst program change statin use in Australia? Med J Aust. 2015:202(11):591-4.

42. Pharmaceutical Benefits Advisory Committee. Streamline authority initiative review. 2008.

43. Box GE, Jenkins GM, Reinsel GC, Ljung GM. Time series analysis: forecasting and control: John Wiley \& Sons; 2015

44. Wahab IA, Pratt NL, Wiese MD, Kalisch LM, Roughead EE. The validity of sequence symmetry analysis (SSA) for adverse drug reaction signal detection. Pharmacoepidemiol Drug Saf. 2013;22(5):496-502.

45. Vitry A, Wong SA, Roughead EE, Ramsay E, Barratt J. Validity of medicationbased co-morbidity indices in the Australian elderly population. Aust N Z J Public Health. 2009:33(2):126-30.

46. Sloan KL, Sales AE, Liu C-F, Fishman P, Nichol P, Suzuki NT, et al. Construction and characteristics of the RxRisk-V: a VA-adapted pharmacybased case-mix instrument. Med Care. 2003;41(6):761-74.

47. Gallego JA, Nielsen J, De Hert M, Kane JM, Correll CU. Safety and tolerability of antipsychotic polypharmacy. Expert Opin Drug Saf. 2012;11(4):527-42.

48. Tsiropoulos I, Andersen M, Hallas J. Adverse events with use of antiepileptic drugs: a prescription and event symmetry analysis. Pharmacoepidemiol Drug Saf. 2009;18(6):483-91.

49. Ho PM, Magid DJ, Shetterly SM, Olson KL, Maddox TM, Peterson PN, et al. Medication nonadherence is associated with a broad range of adverse outcomes in patients with coronary artery disease. Am Heart J. 2008;155(4):772-9.

50. Ramsay EN, Pratt NL, Ryan P, Roughead EE. Proton pump inhibitors and the risk of pneumonia: a comparison of cohort and self-controlled case series designs. BMC Med Res Methodol. 2013;13(1):82.

51. Douglas IJ, Smeeth L. Exposure to antipsychotics and risk of stroke: self controlled case series study. Br Med J. 2008;337:a1227. 
52. Pratt NL, Roughead EE, Ramsay E, Salter A, Ryan P. Risk of hospitalization for stroke associated with antipsychotic use in the elderly. Drugs Aging. 2010; 27(11):885-93.

53. Jackson T. Cost estimates for hospital inpatient care in Australia: evaluation of alternative sources. Aust N Z J Public Health. 2000;24(3):234-41.

54. Levinson W. Searching for effective innovations I: reducing low-value care. Webinar. Washington: Academy of Health; 2015. Available from: http://www. academyhealth.org/Events/events.cfm?ltemNumber=16876. Accessed Jan 2017.

55. Mackenzie A. Balancing the benefits and risks of empirical antibiotics for sinusitis: a teachable moment. JAMA Intern Med. 2014;174(8):1221-2.

56. Lader M. Benzodiazepines revisited—will we ever learn? Addiction. 2011; 106(12):2086-109.

57. Howell MD, Novack V, Grgurich P, Soulliard D, Novack L, Pencina M, et al. latrogenic gastric acid suppression and the risk of nosocomial Clostridium difficile infection. Arch Intern Med. 2010:170(9):784-90.

58. Abraham NS. Proton pump inhibitors: potential adverse effects. Curr Opin Gastroenterol. 2012;28(6):615-20.

59. McKean A, Monasterio E. Off-label use of atypical antipsychotics. CNS Drugs. 2012:26(5):383-90.

60. Dull RB, Friedman SK, Risoldi ZM, Rice EC, Starlin RC, Destache CJ. Antimicrobial treatment of asymptomatic bacteriuria in noncatheterized adults: a systematic review. Pharmacotherapy. 2014;34(9):941-60.

61. Pirmohamed M, James S, Meakin S, Green C, Scott AK, Walley TJ, et al. Adverse drug reactions as cause of admission to hospital: prospective analysis of 18820 patients. Br Med J. 2004;329(7456):15-9.

62. Rahman S, Malcoun A. Nonsteroidal antiinflammatory drugs, cyclooxygenase-2, and the kidneys. Prim Care. 2014;41(4):803-21.

63. Fernandez-Balsells MM, Murad MH, Lane M, Lampropulos JF, Albuquerque F, Mullan RJ, et al. Adverse effects of testosterone therapy in adult men: a systematic review and meta-analysis. J Clin Endocrinol Metabol. 2010;95(6): 2560-75.

64. Choosing Wisely USA. Available from: http://www.choosingwisely.org/. Accessed Jan 2017.

65. Choosing Wisely Canada. Available from: http://www.choosingwiselycanada. org/. Accessed Jan 2017

66. Slow Medicine. Choosing Wisely the Italian Project. Available from: http://www.choosingwiselyitaly.org/index.php/it/. Accessed Apr 2017.

67. Choosing Wisely Australia. Available from: http://www.choosingwisely.org. au/home. Accessed Jan 2017.

68. Choosing Wisely Netherlands Campaign. Available from: http://www. demedischspecialist.nl/file/314/download?token=dewWtajm. Accessed Jan 2017.

69. Smarter Medicine Switzerland. Available from: http://www.smartermedicine. ch/de. Accessed Jan 2017

\section{Submit your next manuscript to BioMed Central and we will help you at every step:}

- We accept pre-submission inquiries

- Our selector tool helps you to find the most relevant journal

- We provide round the clock customer support

- Convenient online submission

- Thorough peer review

- Inclusion in PubMed and all major indexing services

- Maximum visibility for your research

Submit your manuscript at www.biomedcentral.com/submit 